\title{
The impact of COVID-19 telephone consultations on renal clinic non-attendance
}

\author{
Authors: Keith Chan, ${ }^{\mathrm{A}}$ Thomas Cornish ${ }^{\mathrm{A}}$ and Jo Taylor ${ }^{\mathrm{A}}$
}

\section{Introduction}

Non-attendance accounts for $6.4 \%$ of all outpatient appointments. It is a significant financial burden to the NHS (circa $£ 1$ billion annually) and unfairly compromises vital services for other patients. ${ }^{1}$ The COVID-19 pandemic meant classical face-to-face consultations were unfavourable and alternative approaches were required. Given the ever-increasing demands on the NHS, the circumstances of the pandemic provided an opportunity to explore the impact of outpatient non-attendance via different consultation methods. We evaluated non-attendance rates between face to face clinic and telephone consultations in a nephrology clinic.

\section{Materials and methods}

A retrospective analysis compared non-attendance in renal outpatient telephone consultations during the 2020 COVID-19 restriction with a corresponding 15 -week period between April and August of face-to-face clinics in 2018. Telephone non-attendance was defined as failed patient contact despite a pre-arranged appointment. Patient data were derived from four hospital sites and two non-district general hospital dialysis units served by the Dorset Renal Service. Additional data were collected for non-attendees including age, sex, travelling distance, appointment type (new vs follow-up), and if available, pre-clinic renal blood results. The data were analysed using chi-squared and Kruskal-Wallis tests.

\section{Results and discussion}

$264 / 3,397$ (7.8\%) face to face appointments in 232 patients were missed during clinics in 2018 compared to 204/3,341 telephone appointments (6.1\%) missed by 190 patients in 2020. (chisquared with Yates correction 6.9745; $\mathrm{p}=0.008268$ ). Median age of non-attendees was 60 years (range 17-94) in 2018 and 60 years (17-92) in 2020; 147/264 appointments (55.7\%) were missed by male patients in 2018 and 122/204 (59.8\%) in 2020;29/264 missed appointments (11\%) were new patient appointments in 2018 and 14/204 (6.9\%) in 2020; median distance from clinic of non-attendees was 6 miles (range 1-44) in 2018 and 6 miles (1-84) in 2020; 28/232 (12.1\%) patients missed more than one

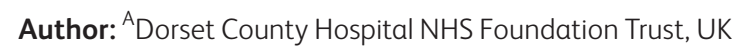

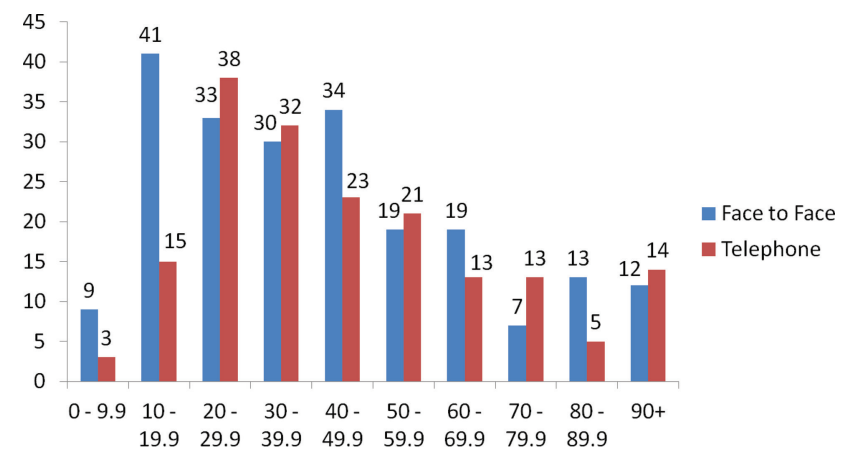

Fig 1. Pre-clinic eGFR in non-attending patients for 2018 face-to-face and corresponding 2020 telephone consultations.

appointment in 2018 and 14/190 (7.4\%) in 2020; 103/264 (39\%) 2018 appointments were missed by patients on renal replacement therapy and 55/204 (27\%) in 2020. 217 pre-clinic blood tests were taken from the 2018 non-attenders, and 177 from the 2020 telephone cohort. The distribution of eGFR results in the two cohorts is represented in Fig 1. Non-parametric KrukalWallis testing demonstrated no difference $(\mathrm{p}=0.094)$ in eGFR distribution between clinic settings. However, 50/217 (23\%) tests prior to the non-attended face to face clinics in 2018 and $18 / 177$ (10.2\%) tests prior to the missed telephone appointments demonstrated an eGFR $<20 \mathrm{~mL} / \mathrm{min} / \mathrm{m}^{2}$ (chi-squared with Yates correction 7.3567; $\mathrm{p}=0.006681$ ).

\section{Conclusion}

The COVID-19 pandemic has provided an opportunity to revise and enhance routine medical care. In this study on renal outpatients, there was a significant albeit small improvement in non-attendee rate with telephone consultation. There were also fewer non-attending patients with advanced chronic kidney disease.

\section{Conflict of Interest}

None declared.

\section{Reference}

1 NHS Digital. Hospital outpatient activity 2018-2019. NHS, 2019. https://digital.nhs.uk/data-and-information/publications/statistical/ hospital-outpatient-activity/2018-19 [Accessed 02 October 2020]. 